\title{
Amenability for dual Banach algebras
}

\author{
by \\ Volker Runde (Edmonton, AB)
}

\begin{abstract}
We define a Banach algebra $\mathfrak{A}$ to be dual if $\mathfrak{A}=\left(\mathfrak{A}_{*}\right)^{*}$ for a closed submodule $\mathfrak{A}_{*}$ of $\mathfrak{A}^{*}$. The class of dual Banach algebras includes all $W^{*}$-algebras, but also all algebras $M(G)$ for locally compact groups $G$, all algebras $\mathcal{L}(E)$ for reflexive Banach spaces $E$, as well as all biduals of Arens regular Banach algebras. The general impression is that amenable, dual Banach algebras are rather the exception than the rule. We confirm this impression. We first show that under certain conditions an amenable dual Banach algebra is already super-amenable and thus finite-dimensional. We then develop two notions of amenability - Connes amenability and strong Connes amenability — which take the $w^{*}$-topology on dual Banach algebras into account. We relate the amenability of an Arens regular Banach algebra $\mathfrak{A}$ to the (strong) Connes amenability of $\mathfrak{A}^{* *}$; as an application, we show that there are reflexive Banach spaces with the approximation property such that $\mathcal{L}(E)$ is not Connes amenable. We characterize the amenability of inner amenable locally compact groups in terms of their algebras of pseudo-measures. Finally, we give a proof of the known fact that the amenable von Neumann algebras are the subhomogeneous ones, which avoids the equivalence of amenability and nuclearity for $C^{*}$-algebras.
\end{abstract}

1. Introduction. Amenable Banach algebras were introduced by B. E. Johnson in [Joh 2], and have since then turned out to be extremely interesting objects of research. The definition of an amenable Banach algebra is strong enough to allow for the development of a rich general theory, but still weak enough to include a variety of interesting examples. Very often, for a class of Banach algebras, the amenability condition singles out an important subclass: For a locally compact group $G$, the convolution algebra $L^{1}(G)$ is amenable if and only if $G$ is amenable in the classical sense ([Joh 2]); a $C^{*}$-algebra is amenable if and only if it is nuclear ([Con 2], [B-P], [Haa]); and a uniform algebra with character space $\Omega$ is amenable if and only if it is $\mathcal{C}_{0}(\Omega)$ ([She]). To determine, for a given class of Banach algebras, which algebras in it are the amenable ones is an active area of research: For instance, it is still open for which Banach spaces $E$ the Banach algebra $\mathcal{K}(E)$ of all compact operators on $E$ is amenable (see $[\mathrm{G}-\mathrm{J}-\mathrm{W}]$ for partial results),

2000 Mathematics Subject Classification: Primary 46H99; Secondary 43A10, 46B28, 46H25, 46L10, 46M20. 
and examples of radical amenable Banach algebras have only recently been given ([Run 1], [Rea]).

In this paper, we consider the following class of Banach algebras:

Definition 1.1. A Banach algebra $\mathfrak{A}$ is said to be dual if there is a closed submodule $\mathfrak{A}_{*}$ of $\mathfrak{A}^{*}$ such that $\mathfrak{A}=\left(\mathfrak{A}_{*}\right)^{*}$.

If $\mathfrak{A}$ is a dual Banach algebra, the predual module $\mathfrak{A}_{*}$ need not be unique. In this paper, however, it is always clear, for a dual Banach algebra $\mathfrak{A}$, to which $\mathfrak{A}_{*}$ we are referring. In particular, we may speak of the $w^{*}$-topology on $\mathfrak{A}$ without ambiguity.

The notion of a dual Banach algebra as defined in Definition 1.1 is by no means universally accepted. The name "dual Banach algebra" occurs in the literature in several contexts - often quite far apart from Definition 1.1. On the other hand, Banach algebras satisfying Definition 1.1 may appear with a different name tag; for instance, dual Banach algebras in our sense are called Banach algebras with (DM) in [C-G 1] and [C-G 2].

We note a few elementary properties of dual Banach algebras:

Proposition 1.2. Let $\mathfrak{A}$ be a dual Banach algebra. Then:

(i) Multiplication in $\mathfrak{A}$ is separately $w^{*}$-continuous.

(ii) $\mathfrak{A}$ has an identity if and only if it has a bounded approximate identity.

(iii) The Dixmier projection $\pi: \mathfrak{A}^{* *} \cong \mathfrak{A}_{*}^{* * *} \rightarrow \mathfrak{A}_{*}^{*} \cong \mathfrak{A}$ is an algebra homomorphism with respect to either Arens multiplication on $\mathfrak{A}^{* *}$.

Proof. (i) and (ii) are obvious, and (iii) follows from (i) and [Pal 1, Theorem 1].

The main reason for us to consider dual Banach algebras is that this class covers a wide range of examples:

EXAmples. 1. Any $W^{*}$-algebra is dual.

2. If $G$ is a locally compact group, then $M(G)$ is dual (with $M(G)_{*}=$ $\left.\mathcal{C}_{0}(G)\right)$.

3. If $E$ is a reflexive Banach space, then $\mathcal{L}(E)$ is dual (with $\mathcal{L}(E)_{*}=$ $\left.E \hat{\otimes} E^{*}\right)$.

4. If $\mathfrak{A}$ is an Arens regular Banach algebra, then $\mathfrak{A}^{* *}$ is dual; in particular, every reflexive Banach algebra is dual.

Comparing this list of dual Banach algebras with our stock of amenable Banach algebras, the overlap is surprisingly small. But although there are few examples of dual Banach algebras which are known to be amenable, there are equally few dual Banach algebras for which we positively know that they are not amenable:

- A $W^{*}$-algebra is amenable if and only if it is subhomogeneous (this follows from [Was, Corollary (1.9)] and [Con 2]; see also [L-L-W]). Even for 
such a "simple" object as $\mathbb{M}_{\infty}:=\ell^{\infty}-\bigoplus_{n=1}^{\infty} \mathbb{M}_{n}$, the proof of non-amenability requires that amenability implies nuclearity.

- If $G$ is a locally compact group, then $M(G)$ is amenable if and only if $G$ is discrete and amenable ([D-G-H]).

- The only Banach spaces $E$ for which $\mathcal{L}(E)$ is known to be amenable are the finite-dimensional ones, and they may well be the only ones. For a Hilbert space $\mathfrak{H}$, the results on amenable von Neumann algebras imply that $\mathcal{L}(\mathfrak{H})$ is not amenable unless $\mathfrak{H}$ is finite-dimensional. It seems to be unknown, however, if $\mathcal{L}\left(\ell^{p}\right)$ is non-amenable for $p \in(1, \infty) \backslash\{2\}$.

- The only known Arens regular Banach algebras $\mathfrak{A}$ for which $\mathfrak{A}^{* *}$ is amenable are the subhomogeneous $C^{*}$-algebras; in particular, no infinitedimensional, reflexive, amenable Banach algebra is known. (It seems that the demand that $\mathfrak{A}^{* *}$ be amenable is very strong: It necessitates $\mathfrak{A}$ to be amenable ([G-L-W], [Gou]) and, for many classes of Banach algebras, forces $\mathfrak{A}$ to be finite-dimensional ([G-R-W], [G-L-W], [Run 1]).)

The general impression thus is that amenability in the sense of [Joh 2] is too strong to allow for the development of a rich theory for dual Banach algebras, and that some notion of amenability taking the $w^{*}$-topology on dual Banach algebras into account is more appropriate ([Grø, Question 10]). Nevertheless, although amenability seems to be a condition which is in conflict with Definition 1.1, this impression is supported by surprisingly few proofs, and even where such proofs exist - in the $W^{*}$-case, for instance-they often seem inappropriately deep.

This paper therefore aims in two directions: First, we want to substantiate our impression that dual Banach algebras are rarely amenable with theorems, and secondly, we want to develop a suitable notion of amenabilitywhich we shall call Connes amenability-for dual Banach algebras.

2. Amenability preliminaries. Let $\mathfrak{A}$ be a Banach algebra, and let $E$ be a Banach $\mathfrak{A}$-bimodule. A derivation from $\mathfrak{A}$ into $E$ is a bounded linear map satisfying

$$
D(a b)=a \cdot D b+(D a) \cdot b \quad(a, b \in \mathfrak{A}) .
$$

As is customary, we write $\mathcal{Z}^{1}(\mathfrak{A}, E)$ for the Banach space of all derivations from $\mathfrak{A}$ into $E$. For $x \in E$, the linear map

$$
\operatorname{ad}_{x}: \mathfrak{A} \rightarrow E, \quad a \mapsto a \cdot x-x \cdot a,
$$

is a derivation. Derivations of this form are called inner derivations; the normed space of all inner derivations from $\mathfrak{A}$ to $E$ is denoted by $\mathcal{B}^{1}(\mathfrak{A}, E)$. The quotient space $\mathcal{H}^{1}(\mathfrak{A}, E):=\mathcal{Z}^{1}(\mathfrak{A}, E) / \mathcal{B}^{1}(\mathfrak{A}, E)$ is called the first cohomology group (or rather: space) of $\mathfrak{A}$ with coefficients in $E$. 
The dual space of a Banach $\mathfrak{A}$-bimodule can be made into a Banach $\mathfrak{A}$-bimodule as well via

$$
\langle x, a \cdot \phi\rangle:=\langle x \cdot a, \phi\rangle, \quad\langle x, \phi \cdot a\rangle:=\langle a \cdot x, \phi\rangle \quad\left(a \in \mathfrak{A}, \phi \in E^{*}, x \in E\right) .
$$

A Banach algebra $\mathfrak{A}$ is defined to be amenable if $\mathcal{H}^{1}\left(\mathfrak{A}, E^{*}\right)=\{0\}$ for every Banach $\mathfrak{A}$-bimodule $E$ ([Joh 2]). References for amenable Banach algebras are [Joh 2] as well as [Hel 1], where a different, but equivalent approach, based on the notion of flatness in topological homology, is given.

We shall also require a characterization of amenable Banach algebras in terms of approximate diagonals as given in [Joh 3]. Let $\mathfrak{A} \hat{\otimes} \mathfrak{A}$ denote the projective tensor product of $\mathfrak{A}$ with itself. Then $\mathfrak{A} \hat{\otimes} \mathfrak{A}$ is a Banach A-bimodule through

$$
a \cdot(x \otimes y):=a x \otimes y \quad \text { and } \quad(x \otimes y) \cdot a:=x \otimes y a \quad(a, x, y \in \mathfrak{A}) .
$$

Let $\Delta: \mathfrak{A} \hat{\otimes} \mathfrak{A} \rightarrow \mathfrak{A}$ be the multiplication operator, i.e. $\Delta(a \otimes b):=a b$ for $a, b \in \mathfrak{A}$ (sometimes, when we wish to emphasize the algebra $\mathfrak{A}$, we also write $\left.\Delta_{\mathfrak{A}}\right)$. An approximate diagonal for $\mathfrak{A}$ is a bounded net $\left(m_{\alpha}\right)_{\alpha}$ in $\mathfrak{A} \hat{\otimes} \mathfrak{A}$ such that

$$
a \cdot m_{\alpha}-m_{\alpha} \cdot a \rightarrow 0 \quad \text { and } \quad a \Delta m_{\alpha} \rightarrow a \quad(a \in \mathfrak{A}) .
$$

The algebra $\mathfrak{A}$ is amenable if and only if it has an approximate diagonal ([Joh 3]).

There are several variants of amenability, two of which will be discussed here: super-amenability and Connes amenability.

A Banach algebra $\mathfrak{A}$ is said to be super-amenable (or contractible) if $\mathcal{H}^{1}(\mathfrak{A}, E)=\{0\}$ for every Banach $\mathfrak{A}$-bimodule $E$. Equivalently, $\mathfrak{A}$ is superamenable if it has a diagonal, i.e. a constant approximate diagonal ([C-L, Theorem 6.1]). All algebras $\mathbb{M}_{n}$ with $n \in \mathbb{N}$ and all finite direct sums of such algebras are super-amenable; no other examples are known. Conversely, it is known that every super-amenable Banach algebra $\mathfrak{A}$ which satisfies some rather mild hypotheses in terms of Banach space geometry must be a finite direct sum of full matrix algebras ([Sel], for example; see [Run 1] for a survey and some refinements). In particular, every super-amenable Banach algebra $\mathfrak{A}$ with the approximation property is of the form

$$
\mathfrak{A} \cong \mathbb{M}_{n_{1}} \oplus \ldots \oplus \mathbb{M}_{n_{k}}
$$

with $n_{1}, \ldots, n_{k} \in \mathbb{N}$.

In $[\mathrm{J}-\mathrm{K}-\mathrm{R}]$, B. E. Johnson, R. V. Kadison, and J. Ringrose introduced a notion of amenability for von Neumann algebras which takes the ultraweak topology into account. The basic concepts, however, make sense for arbitrary dual Banach algebras. Let $\mathfrak{A}$ be a dual Banach algebra, and let $E$ be a Banach $\mathfrak{A}$-bimodule. Then we call $E^{*}$ a $w^{*}$-Banach $\mathfrak{A}$-bimodule if, for each 
$\phi \in E^{*}$, the maps

$$
\mathfrak{A} \rightarrow E^{*}, \quad a \mapsto\left\{\begin{array}{l}
a \cdot \phi, \\
\phi \cdot a,
\end{array}\right.
$$

are $w^{*}$-continuous. We write $\mathcal{Z}_{w^{*}}^{1}\left(\mathfrak{A}, E^{*}\right)$ for the $w^{*}$-continuous derivations from $\mathfrak{A}$ into $E^{*}$. The $w^{*}$-continuity of the maps (1) implies that $\mathcal{B}^{1}\left(\mathfrak{A}, E^{*}\right) \subset$ $\mathcal{Z}_{w^{*}}^{1}\left(\mathfrak{A}, E^{*}\right)$, so that $\mathcal{H}_{w^{*}}^{1}\left(\mathfrak{A}, E^{*}\right):=\mathcal{Z}_{w^{*}}^{1}\left(\mathfrak{A}, E^{*}\right) / \mathcal{B}^{1}\left(\mathfrak{A}, E^{*}\right)$ is a meaningful definition.

Definition 2.1. A dual Banach algebra $\mathfrak{A}$ is Connes amenable if $\mathcal{H}_{w^{*}}^{1}\left(\mathfrak{A}, E^{*}\right)=\{0\}$ for every $w^{*}$-Banach $\mathfrak{A}$-bimodule $E^{*}$.

REMARKS. 1. Although the notion of Connes amenability was introduced in $[\mathrm{J}-\mathrm{K}-\mathrm{R}]$ (for $W^{*}$-algebras), it is most commonly associated with A. Connes' paper [Con 1]; this motivates our choice of terminology (compare also [Hel 2]).

2. Definition 2.1 is a special case of a notion of amenability introduced in $[\mathrm{C}-\mathrm{G} 1]$. There, for an arbitrary Banach algebra $\mathfrak{A}$ and a submodule $\Phi$ of $\mathfrak{A}^{*}$ satisfying certain properties, $\Phi$-amenability is defined. If $\mathfrak{A}$ is a dual Banach algebra, then $\mathfrak{A}_{*}$ satisfies all the requirements for $\Phi$ in $[\mathrm{C}-\mathrm{G} 1]$, and $\mathfrak{A}$ is Connes amenable if and only if it is $\mathfrak{A}_{*}$-amenable in the sense of [C-G 1 ].

3. The rôle of the Radon-Nikodým property. Let $\mathfrak{A}$ be a dual Banach algebra, and let $\mathfrak{A}_{*}$ be its predual as in Definition 1.1. Let $\mathfrak{A}_{*} \check{\otimes} \mathfrak{A}_{*}$ be the injective tensor product of $\mathfrak{A}_{*}$ with itself. Then we have a canonical map from $\mathfrak{A} \hat{\otimes} \mathfrak{A}$ into $\left(\mathfrak{A}_{*} \check{\otimes} \mathfrak{A}_{*}\right)^{*}$, which has closed range if $\mathfrak{A}$ has the bounded approximation property ([D-F, 16.3, Corollary 2]).

If $\mathfrak{A}$ is amenable, a naive approach to show that $\mathfrak{A}$ is super-amenable would be as follows:

STEP 1. Let $\left(m_{\alpha}\right)_{\alpha}$ be an approximate diagonal for $\mathfrak{A}$, and choose an accumulation point $m$ of $\left(m_{\alpha}\right)_{\alpha}$ in the topology induced by $\mathfrak{A}_{*} \check{\otimes} \mathfrak{A}_{*}$.

STEP 2. Show that $m$ is a diagonal for $\mathfrak{A}$.

There are problems in both steps (and since there are amenable, dual Banach algebras which are not super-amenable this is no surprise). In Step 1 , the main problem is that the accumulation point $m \in\left(\mathfrak{A}_{*} \check{\otimes} \mathfrak{A}_{*}\right)^{*}$ need not lie in $\mathfrak{A} \hat{\otimes} \mathfrak{A}$. In view of [D-F, 16.6, Theorem], it is clear that in order to make Step 1 work, we have to require the Radon-Nikodým property for $\mathfrak{A}$.

We have the following theorem:

THEOREM 3.1. Let $\mathfrak{A}$ be an amenable dual Banach algebra having both the approximation property and the Radon-Nikodym property. Suppose further that there is a family $\left(I_{\lambda}\right)_{\lambda}$ of $w^{*}$-closed ideals of $\mathfrak{A}$, each with finite codimension, such that $\bigcap_{\lambda} I_{\lambda}=\{0\}$. Then there are $n_{1}, \ldots, n_{k} \in \mathbb{N}$ such 
that

$$
\mathfrak{A} \cong \mathbb{M}_{n_{1}} \oplus \ldots \oplus \mathbb{M}_{n_{k}}
$$

Proof. Let $\mathfrak{A}_{*}$ denote the predual of $\mathfrak{A}$. Since $\mathfrak{A}$ has both the approximation property and the Radon-Nikodým property, we have

$$
\mathfrak{A} \hat{\otimes} \mathfrak{A} \cong\left(\mathfrak{A}_{*} \check{\otimes} \mathfrak{A}_{*}\right)^{*}
$$

by [D-F, 16.6, Theorem]. We thus have a natural $w^{*}$-topology on $\mathfrak{A} \hat{\otimes} \mathfrak{A}$. Let $\left(m_{\alpha}\right)_{\alpha}$ be an approximate diagonal for $\mathfrak{A}$, and let $m \in \mathfrak{A} \hat{\otimes} \mathfrak{A}$ be a $w^{*}$-accumulation point of $\left(m_{\alpha}\right)_{\alpha}$; passing to a subnet we can assume that $m=w^{*}-\lim _{\alpha} m_{\alpha}$.

We claim that $m$ is a diagonal for $\mathfrak{A}$. It is clear that $m \in \mathcal{Z}^{0}(\mathfrak{A}, \mathfrak{A} \hat{\otimes} \mathfrak{A})$, so all we have to show is that $\Delta m=e_{\mathfrak{A}}$. Let $\pi_{\lambda}: \mathfrak{A} \rightarrow \mathfrak{A} / I_{\lambda}$ be the canonical epimorphism. Since $I_{\lambda}$ is $w^{*}$-closed, each quotient algebra $\mathfrak{A} / I_{\lambda}$ is again dual with the predual

$$
{ }^{\perp} I_{\lambda}=\left\{\phi \in \mathfrak{A}_{*}:\langle\phi, a\rangle=0 \text { for all } a \in I_{\lambda}\right\} .
$$

Let $\iota_{\lambda}:{ }^{\perp} I_{\lambda} \rightarrow \mathfrak{A}_{*}$ be the inclusion map. Then $\pi_{\lambda} \otimes \pi_{\lambda}: \mathfrak{A} \hat{\otimes} \mathfrak{A} \rightarrow \mathfrak{A} / I_{\lambda} \hat{\otimes}$ $\mathfrak{A} / I_{\lambda}$ is the tranpose of $\iota_{\lambda} \otimes \iota_{\lambda}:{ }^{\perp} I_{\lambda} \check{\otimes}{ }^{\perp} I_{\lambda} \rightarrow \mathfrak{A}_{*} \check{\otimes} \mathfrak{A}_{*}$ (since $I_{\lambda}$ has finite codimension, we clearly have $\left.\mathfrak{A} / I_{\lambda} \hat{\otimes} \mathfrak{A} / I_{\lambda} \cong\left({ }^{\perp} I_{\lambda} \check{\otimes}{ }^{\perp} I_{\lambda}\right)^{*}\right)$. Thus, $\pi_{\lambda} \otimes \pi_{\lambda}$ is $w^{*}$-continuous, so that

$$
\left(\pi_{\lambda} \otimes \pi_{\lambda}\right) m=w^{*}-\lim _{\alpha}\left(\pi_{\lambda} \otimes \pi_{\lambda}\right) m_{\alpha}
$$

Since $\mathfrak{A} / I_{\lambda} \hat{\otimes} \mathfrak{A} / I_{\lambda}$ is finite-dimensional, there is only one vector space topology on it; in particular, $\left(\pi_{\lambda} \otimes \pi_{\lambda}\right) d$ is the norm limit of $\left(\left(\pi_{\lambda} \otimes \pi_{\lambda}\right) d_{\alpha}\right)_{\alpha}$. Since

$$
\Delta_{\mathfrak{A} / I_{\lambda}} \circ\left(\pi_{\lambda} \otimes \pi_{\lambda}\right)=\pi_{\lambda} \circ \Delta_{\mathfrak{A}}
$$

we obtain

$$
\left(\pi_{\lambda} \circ \Delta_{\mathfrak{A}}\right) m=\lim _{\alpha}\left(\Delta_{\mathfrak{A} / I_{\lambda}} \circ\left(\pi_{\lambda} \otimes \pi_{\lambda}\right)\right) m_{\alpha}=e_{\mathfrak{A} / I_{\lambda}}
$$

Since $\left(\pi_{\lambda}\right)_{\lambda}$ separates the points of $\mathfrak{A}$, it follows that $\Delta_{\mathfrak{A}} m=e_{\mathfrak{A}}$. Hence, $m$ is a diagonal for $\mathfrak{A}$.

REMARK. It is essential for Theorem 3.1 to hold that $\mathfrak{A}$ has the RadonNikodým property. For example, the algebra $\ell^{\infty}$ is an amenable dual Banach algebra which has a family $\left(I_{\lambda}\right)_{\lambda}$ of $w^{*}$-closed ideals as in Theorem 3.1 , but is infinite-dimensional.

Since separable dual spaces as well as reflexive Banach spaces automatically have the Radon-Nikodým property ([D-F, D3]), we obtain the following corollaries, the first of which is a nice dichotomy and the second improves [G-R-W, Corollary 2.3] and is related to [Run 1, Proposition 2.3]:

COROLlary 3.2. Let $\mathfrak{A}$ be an amenable dual Banach algebra having the approximation property. Suppose further that there is a family $\left(I_{\lambda}\right)_{\lambda}$ of $w^{*}-$ 
closed ideals of $\mathfrak{A}$, each with finite codimension, such that $\bigcap_{\lambda} I_{\lambda}=\{0\}$. Then one of the following holds:

(i) $\mathfrak{A}$ is not separable;

(ii) there are $n_{1}, \ldots, n_{k} \in \mathbb{N}$ such that

$$
\mathfrak{A} \cong \mathbb{M}_{n_{1}} \oplus \ldots \oplus \mathbb{M}_{n_{k}} .
$$

Corollary 3.3. Let $\mathfrak{A}$ be an amenable reflexive Banach algebra having the approximation property. Suppose further that there is a family $\left(I_{\lambda}\right)_{\lambda}$ of closed ideals of $\mathfrak{A}$, each with finite codimension, such that $\bigcap_{\lambda} I_{\lambda}=\{0\}$. Then there are $n_{1}, \ldots, n_{k} \in \mathbb{N}$ such that

$$
\mathfrak{A} \cong \mathbb{M}_{n_{1}} \oplus \ldots \oplus \mathbb{M}_{n_{k}}
$$

REMARK. In Corollary 3.3 we can replace the hypothesis that there is a family $\left(I_{\lambda}\right)_{\lambda}$ of closed ideals of $\mathfrak{A}$, each with finite codimension, such that $\bigcap_{\lambda} I_{\lambda}=\{0\}$ by a weaker one. If we assume that the almost periodic functionals on $\mathfrak{A}$ separate points, we still get the same conclusion (this is proved in the same way as [Run 1, Proposition 3.1]). For examples of almost periodic functionals that do not arise from finite-dimensional quotients, see [D- $\ddot{\mathrm{U}}]$.

4. Connes amenability of biduals. In this section, we investigate how, for an Arens regular Banach algebra $\mathfrak{A}$, the amenability of $\mathfrak{A}$ and the Connes amenability of $\mathfrak{A}^{* *}$ are related.

We begin our discussion with some elementary propositions.

Proposition 4.1. Let $\mathfrak{A}$ be a Connes amenable dual Banach algebra. Then $\mathfrak{A}$ has an identity.

Proof. Let $A$ be the Banach $\mathfrak{A}$-bimodule whose underlying linear space is $\mathfrak{A}$ equipped with the following module operations:

$$
a \cdot x:=a x \quad \text { and } \quad x \cdot a:=0 \quad(a, x \in \mathfrak{A}) .
$$

Obviously, $A$ is a $w^{*}$-Banach $\mathfrak{A}$-bimodule, and the identity map from $\mathfrak{A}$ into $A$ is a $w^{*}$-continuous derivation. Since $\mathcal{H}_{w^{*}}^{1}(\mathfrak{A}, A)=\{0\}$, this means that $\mathfrak{A}$ has a right identity. Analogously, one sees that $\mathfrak{A}$ also has a left identity.

Proposition 4.2. Let $\mathfrak{A}$ be a Banach algebra, let $\mathfrak{B}$ be a dual Banach algebra, and let $\theta: \mathfrak{A} \rightarrow \mathfrak{B}$ be a continuous homomorphism with $w^{*}$-dense range. Then:

(i) If $\mathfrak{A}$ is amenable, then $\mathfrak{B}$ is Connes amenable.

(ii) If $\mathfrak{A}$ is dual and Connes amenable, and if $\theta$ is $w^{*}$-continuous, then $\mathfrak{B}$ is Connes amenable.

Proof. Immediate from the definitions. 
Corollary 4.3. Let $\mathfrak{A}$ be an Arens regular Banach algebra. Then, if $\mathfrak{A}$ is amenable, $\mathfrak{A}^{* *}$ is Connes amenable.

If $\mathfrak{A}$ is a $C^{*}$-algebra, then the converse of Corollary 4.3 holds: If $\mathfrak{A}^{* *}$ is Connes amenable, then $\mathfrak{A}$ is amenable ([Con 1], [B-P], [Haa], [Eff], [E-K]). This is a deep, specifically $C^{*}$-algebraic result, for which no analogue in the general Banach algebra setting is available (yet). Under certain circumstances, however, a converse of Corollary 4.3 holds for general Banach algebras:

THEOREM 4.4. Let $\mathfrak{A}$ be an Arens regular Banach algebra which is an ideal in $\mathfrak{A}^{* *}$. Then the following are equivalent:

(i) $\mathfrak{A}$ is amenable.

(ii) $\mathfrak{A}^{* *}$ is Connes amenable.

Proof. Since $\mathfrak{A}^{* *}$ is Connes amenable, it has an identity by Proposition 4.1. By [Pal 2, Proposition 5.1.8], this means that $\mathfrak{A}$ has a bounded approximate identity, $\left(e_{\alpha}\right)_{\alpha}$ say. By [Joh 2], it is therefore sufficient for $\mathfrak{A}$ to be amenable that $\mathcal{H}^{1}\left(\mathfrak{A}, E^{*}\right)=\{0\}$ for each essential Banach $\mathfrak{A}$-bimodule.

Let $E$ be an essential Banach $\mathfrak{A}$-bimodule, and let $D: \mathfrak{A} \rightarrow E^{*}$ be a derivation. The following construction is carried out in [Joh 2] with the double centralizer algebra instead of $\mathfrak{A}^{* *}$, but an inspection of the argument there shows that it carries over to our situation. Since $E$ is essential, there are, for each $x \in E$, elements $b, c \in \mathfrak{A}$ and $y, z \in E$ with $x=b \cdot y=z \cdot c$. Define an $\mathfrak{A}$-bimodule action of $\mathfrak{A}^{* *}$ on $E$ by letting

$$
a \cdot(b \cdot y):=a b \cdot y, \quad(z \cdot c) \cdot a:=z \cdot c a \quad\left(a \in \mathfrak{A}^{* *}, b, c \in \mathfrak{A}, y, z \in E\right) .
$$

It can be shown that this module action is well defined and turns $E$ into a Banach $\mathfrak{A}^{* *}$-bimodule. Consequently, $E^{*}$ equipped with the corresponding dual module action is a Banach $\mathfrak{A}^{* *}$-bimodule as well.

We claim that $E^{*}$ is even a $w^{*}$-Banach $\mathfrak{A}^{* *}$-bimodule. Let $\left(a_{\beta}\right)_{\beta}$ be a net in $\mathfrak{A}^{* *}$ such that $a_{\beta} \stackrel{w^{*}}{\longrightarrow} 0$, let $\phi \in E^{*}$, and let $x \in E$. Let $b \in \mathfrak{A}$ and $y \in E$ be such that $x=y \cdot b$. Since the $w^{*}$-topology of $\mathfrak{A}^{* *}$ restricted to $\mathfrak{A}$ is the weak topology, we have $b a_{\beta} \stackrel{w}{\longrightarrow} 0$, so that

$$
x \cdot a_{\beta}=y \cdot b a_{\beta} \stackrel{w}{\longrightarrow} 0
$$

and consequently

$$
\left\langle x, a_{\beta} \cdot \phi\right\rangle=\left\langle x \cdot a_{\beta}, \phi\right\rangle \rightarrow 0 .
$$

Since $x \in E$ was arbitrary, this means that $a_{\beta} \cdot \phi \stackrel{w^{*}}{\longrightarrow} 0$. Analogously, one shows that $\phi \cdot a_{\beta} \stackrel{w^{*}}{\longrightarrow} 0$.

Following [Joh 2] again, we define a derivation $\widetilde{D}: \mathfrak{A}^{* *} \rightarrow E^{*}$ by letting

$$
\widetilde{D} a=w^{*}-\lim _{\alpha}\left[D\left(a e_{\alpha}\right)-a \cdot D e_{\alpha}\right]
$$


We claim that $\widetilde{D} \in \mathcal{Z}_{w^{*}}^{1}\left(\mathfrak{A}^{* *}, E^{*}\right)$. Let again $\left(a_{\beta}\right)_{\beta}$ be a net in $\mathfrak{A}^{* *}$ such that $a_{\beta} \stackrel{w^{*}}{\longrightarrow} 0$, let $x \in E$, and let $b \in \mathfrak{A}$ and $y \in E$ be such that $x=b \cdot y$. Then we have

$$
\left\langle x, \widetilde{D} a_{\beta}\right\rangle=\left\langle b \cdot y, \widetilde{D} a_{\beta}\right\rangle=\left\langle y,\left(\widetilde{D} a_{\beta}\right) \cdot b\right\rangle=\left\langle y, D\left(a_{\beta} b\right)-a_{\beta} \cdot D b\right\rangle \rightarrow 0,
$$

since $D$ is weakly continuous and $E^{*}$ is a $w^{*}$-Banach $\mathfrak{A}^{* *}$-bimodule.

From the Connes amenability of $\mathfrak{A}^{* *}$ we conclude that $\widetilde{D}$, and hence $D$, is inner.

REMARK. In [Gou], F. Gourdeau showed that, whenever $\mathfrak{A}$ is a Banach algebra, $E$ is a Banach $\mathfrak{A}$-bimodule, and $D: \mathfrak{A} \rightarrow E$ is a derivation, there is an $\mathfrak{A}^{* *}$-bimodule action on $E^{* *}$ turning $D^{* *}: \mathfrak{A}^{* *} \rightarrow E^{* *}$ into a (necessarily $w^{*}$-continuous) derivation. However, even if $E$ is a dual Banach $\mathfrak{A}$-bimodule, there is no need for $E^{* *}$ to be a $w^{*}$-Banach $\mathfrak{A}$-bimodule, so that, in general, we cannot draw any conclusion on the amenability of $\mathfrak{A}$ from the Connes amenability of $\mathfrak{A}^{* *}$.

By $\left[\mathrm{G}-\mathrm{J}-\mathrm{W}\right.$, Theorem 6.9], the space $\ell^{p} \oplus \ell^{q}$ with $p, q \in(1, \infty) \backslash\{2\}$ and $p \neq q$ has the property that $\mathcal{K}\left(\ell^{p} \oplus \ell^{q}\right)$ is not amenable. Hence, Theorem 4.4 yields:

Corollary 4.5. Let $p, q \in(1, \infty) \backslash\{2\}, p \neq q$. Then $\mathcal{L}\left(\ell^{p} \oplus \ell^{q}\right)$ is not Connes amenable.

Proof. Since $\mathcal{K}\left(\ell^{p} \oplus \ell^{q}\right)^{* *} \cong \mathcal{L}\left(\ell^{p} \oplus \ell^{q}\right)$, and since $\mathcal{K}\left(\ell^{p} \oplus \ell^{q}\right)$ is not amenable, $\mathcal{L}\left(\ell^{p} \oplus \ell^{q}\right)$ is not Connes amenable by Theorem 4.4.

Let $\mathfrak{A}$ be a dual Banach algebra, and let $E$ be a Banach $\mathfrak{A}$-bimodule. Then we call an element $\phi \in E^{*}$ a $w^{*}$-element if the maps (1) are $w^{*}$-continuous.

Definition 4.6. A dual Banach algebra with identity $\mathfrak{A}$ is called strongly Connes amenable if, for each each unital Banach $\mathfrak{A}$-bimodule $E$, every $w^{*}$-continuous derivation $D: \mathfrak{A} \rightarrow E^{*}$ whose range consists of $w^{*}$-elements is inner.

We shall give an intrinsic characterization of strongly Connes amenable dual Banach algebras similar to the one given in [Joh 3] for amenable Banach algebras. Recall a few definitions from [C-G 1] (with a different notation, however). Let $\mathfrak{A}$ be a dual Banach algebra with identity, and let $\mathcal{L}_{w^{*}}^{2}(\mathfrak{A}, \mathbb{C})$ be the space of separately $w^{*}$-continuous bilinear functionals on $\mathfrak{A}$. Clearly, $\mathcal{L}_{w^{*}}^{2}(\mathfrak{A}, \mathbb{C})$ is a Banach $\mathfrak{A}$-submodule of $\mathcal{L}^{2}(\mathfrak{A}, \mathbb{C}) \cong(\mathfrak{A} \hat{\otimes} \mathfrak{A})^{*}$. Define

$$
\left(\mathfrak{A} \hat{\otimes}_{w^{*}} \mathfrak{A}\right)^{* *}:=\mathcal{L}_{w^{*}}^{2}(\mathfrak{A}, \mathbb{C})^{*} .
$$

Note that the notation $\left(\mathfrak{A} \hat{\otimes}_{w^{*}} \mathfrak{A}\right)^{* *}$ is merely symbolic: in general, $\left(\mathfrak{A} \hat{\otimes}_{w^{*}} \mathfrak{A}\right)^{* *}$ is not a bidual space. There is a canonical embedding of the algebraic tensor product $\mathfrak{A} \otimes \mathfrak{A}$ into $\left(\mathfrak{A} \hat{\otimes}_{w^{*}} \mathfrak{A}\right)^{* *}$, so that we may identify $\mathfrak{A} \otimes \mathfrak{A}$ with a submodule of $\left(\mathfrak{A} \hat{\otimes}_{w^{*}} \mathfrak{A}\right)^{* *}$. It is immediate that $\mathfrak{A} \otimes \mathfrak{A}$ consists of $w^{*}$-elements 
of $\left(\mathfrak{A} \hat{\otimes}_{w^{*}} \mathfrak{A}\right)^{* *}$. Since multiplicaton in a dual Banach algebra is separately $w^{*}$-continuous, we have

$$
\Delta^{*} \mathfrak{A}_{*} \subset \mathcal{L}_{w^{*}}^{2}(\mathfrak{A}, \mathbb{C}),
$$

so that the multiplication operator $\Delta$ on $\mathfrak{A} \otimes \mathfrak{A}$ extends to $\left(\mathfrak{A} \hat{\otimes}_{w^{*}} \mathfrak{A}\right)^{* *}$; we shall denote this extension by $\Delta_{w^{*}}^{*}$. A virtual $w^{*}$-diagonal for $\mathfrak{A}$ (in

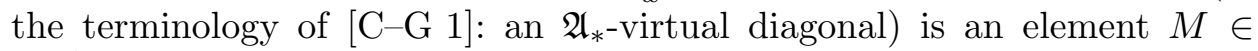
$\left(\mathfrak{A} \hat{\otimes}_{w^{*}} \mathfrak{A}\right)^{* *}$ such that

$$
a \cdot M=M \cdot a \quad(a \in \mathfrak{A}) \quad \text { and } \quad \Delta_{w^{*}}^{* *} M=e_{\mathfrak{A}} .
$$

In $[\mathrm{C}-\mathrm{G}$ 1], G. Corach and J. E. Galé showed that a dual Banach algebra with a virtual $w^{*}$-diagonal is necessarily Connes amenable, and wondered if the converse was also true. For strong Connes amenability, the corresponding question is easy to answer:

Theorem 4.7. For a dual Banach algebra $\mathfrak{A}$, the following are equivalent:

(i) $\mathfrak{A}$ has a virtual $w^{*}$-diagonal.

(ii) $\mathfrak{A}$ is strongly Connes amenable.

Proof. On [C-G 1, p. 90], it is shown that (i) implies the Connes amenability of $\mathfrak{A}$ (the argument for von Neumann algebras from [Eff] carries over verbatim). A closer inspection of the argument given there, however, shows that we already obtain strong Connes amenability.

For the converse, consider the derivation $\operatorname{ad}_{e_{\mathfrak{A}} \otimes e_{\mathfrak{A}}}$. Then, clearly, $\operatorname{ad}_{e_{\mathfrak{A}} \otimes e_{\mathfrak{A}}}$ attains its values at the $w^{*}$-elements of $\operatorname{ker} \Delta_{w^{*}}^{* *}$. By the definition of strong Connes amenability, there is $N \in \operatorname{ker} \Delta_{w^{*}}^{* *} \operatorname{such}$ that $\operatorname{ad}_{N}=\operatorname{ad}_{e_{\mathfrak{A}} \otimes e_{\mathfrak{A}}}$. It follows that $D:=e_{\mathfrak{A}} \otimes e_{\mathfrak{A}}-N$ is a virtual $w^{*}$-diagonal for $\mathfrak{A}$.

REMARK. In [Eff], E. G. Effros proves that a von Neumann algebra is Connes amenable if and only if it has a virtual $w^{*}$-diagonal. Hence, von Neumann algebras are Connes amenable if and only if they are strongly Connes amenable.

For certain Banach algebras $\mathfrak{A}$, the strong Connes amenability of $\mathfrak{A}^{* *}$ entails the amenability of $\mathfrak{A}$ :

TheOREm 4.8. Let $\mathfrak{A}$ be a Banach algebra with the following properties:

(i) every bounded linear map from $\mathfrak{A}$ to $\mathfrak{A}^{*}$ is weakly compact;

(ii) $\mathfrak{A}^{* *}$ is strongly Connes amenable.

Then $\mathfrak{A}$ is amenable.

Proof. By [Gro, Satz 1.29], condition (i) implies (and is, in fact, equivalent to) that every bounded bilinear map from $\mathfrak{A} \times \mathfrak{A}$ into any Banach space is Arens regular; in particular, it ensures that $\mathfrak{A}^{* *}$ is indeed a dual Banach 
algebra. It is thus an immediate consequence of (i) that

$$
(\mathfrak{A} \hat{\otimes} \mathfrak{A})^{* *} \cong\left(\mathfrak{A}^{* *} \hat{\otimes}_{w^{*}} \mathfrak{A}^{* *}\right)^{* *}
$$

as Banach $\mathfrak{A}$-bimodules. Since $\mathfrak{A}^{* *}$ has a virtual $w^{*}$-diagonal by Theorem 4.7, the isomorphism (2) ensures the existence of a virtual diagonal for $\mathfrak{A}$. Thus, $\mathfrak{A}$ is amenable.

ExAmPlES. 1. Every $C^{*}$-algebra satisfies Theorem 4.8(i).

2. Let $E$ be a reflexive Banach space with an unconditional basis. It is implicitly proved in [Ülg] (although not explicitly stated) that $\mathcal{K}(E)$ satisfies Theorem 4.8(i).

5. Dual Banach algebras associated with locally compact groups. As was shown by A. Connes ([Con 2]), Connes amenable von Neumann algebras (with separable predual) are injective. An alternative proof for this is given in $[\mathrm{B}-\mathrm{P}]$. We now give an analogue - in the spirit of [C-G 1, Proposition 2.2] and [C-G 2, Proposition 2.2] - for arbitrary Connes amenable dual Banach algebras.

If $S$ is any subset of an algebra $\mathfrak{B}$, we use $Z_{\mathfrak{B}}(S)$ to denote the centralizer of $S$ in $\mathfrak{B}$, i.e.

$$
Z_{\mathfrak{B}}(S):=\{b \in \mathfrak{B}: b s=s b \text { for all } s \in S\} .
$$

In case $\mathfrak{B}=\mathcal{L}(E)$ for some Banach space $E$, we also write $S^{\prime}$ instead of $Z_{\mathfrak{B}}(S)$. Recall (from $[\mathrm{B}-\mathrm{P}]$ or $[\mathrm{C}-\mathrm{G} 2]$ ) the definition of a quasi-expectation: If $\mathfrak{B}$ is a Banach algebra, and if $\mathfrak{A}$ is a closed subalgebra of $\mathfrak{B}$, a quasiexpectation is a bounded projection $\mathcal{Q}: \mathfrak{B} \rightarrow \mathfrak{A}$ satisfying

$$
\mathcal{Q}(a x b)=a(\mathcal{Q} x) b \quad(a, b \in \mathfrak{A}, x \in \mathfrak{B}) .
$$

Note that we do not require $\|\mathcal{Q}\|=1$.

TheOREM 5.1. Let $\mathfrak{A}$ be a Connes amenable dual Banach algebra, let $\mathfrak{B}$ be a dual Banach algebra with identity, and let $\theta: \mathfrak{A} \rightarrow \mathfrak{B}$ be a unital $w^{*}$-continuous homomorphism. Then there is a quasi-expectation $\mathcal{Q}: \mathfrak{B} \rightarrow$ $Z_{\mathfrak{B}}(\theta(\mathfrak{A}))$.

Proof. More or less a verbatim copy of the proof of [B-P, Theorem 3].

In this section, we will use Theorem 5.1 to characterize the Connes amenability of some dual Banach algebras which arise naturally in abstract harmonic analysis.

For non-discrete abelian $G$, it has long been known that there are nonzero point derivations on $M(G)$ ([B-M]), so that $M(G)$ cannot be amenable. In $[\mathrm{L}-\mathrm{L}]$, A. T.-M. Lau and R. J. Loy started investigating the amenability of $M(G)$ for certain non-abelian $G$. In particular, they were able to show that, for connected $G$, the algebra $M(G)$ is amenable only if $G=\{e\}([\mathrm{L}-\mathrm{L}$, 
Theorem 2.4]). Ultimately, H. G. Dales, F. Ghahramani, and A. Ya. Helemskiı ([D-G-H]) proved: The measure algebra $M(G)$ is amenable if and only if $G$ is discrete so that $M(G)=\ell^{1}(G)=L^{1}(G)$-and amenable.

The picture is completely different for Connes amenability. We have, for example:

Proposition 5.2. Let $G$ be a compact group. Then $M(G)$ is strongly Connes amenable.

Proof. By Theorem 4.7, it is sufficient to construct a virtual $w^{*}$-diagonal for $M(G)$.

For $\phi \in \mathcal{L}_{w^{*}}^{2}(M(G), \mathbb{C})$, define $\bar{\phi}: G \times G \rightarrow \mathbb{C}$ and $\widetilde{\phi}: G \rightarrow \mathbb{C}$ through

$$
\bar{\phi}(x, y):=\phi\left(\delta_{x}, \delta_{y}\right) \quad \text { and } \quad \widetilde{\phi}(x):=\bar{\phi}\left(x, x^{-1}\right) \quad(x, y \in G) \text {. }
$$

Then $\bar{\phi}$ is separately continuous on $G \times G$ and thus belongs to $L^{\infty}(G \times G$, $\mu \times \nu$ ) for any $\nu, \mu \in M(G)$ ([Joh 1]). Since (normalized) Haar measure belongs to $M(G)$, this implies that $\widetilde{\phi} \in L^{\infty}(G) \subset L^{1}(G)$.

Let $m$ denote normalized Haar measure on $G$, and define a functional $M \in\left(M(G) \hat{\otimes}_{w^{*}} M(G)\right)^{* *}$ via

$$
\langle\phi, M\rangle:=\int_{G} \widetilde{\phi}(x) d m(x) \quad\left(\phi \in \mathcal{L}_{w^{*}}^{2}(M(G), \mathbb{C})\right) .
$$

It is routinely checked that $\Delta_{w^{*}}^{* *} M=\delta_{e}$. Let $\mu \in M(G)$, and let $\phi \in$ $\mathcal{L}_{w^{*}}^{2}(M(G), \mathbb{C})$. Then we have:

$$
\begin{aligned}
\langle\phi, \mu \cdot M\rangle & =\langle\phi \cdot \mu, M\rangle \\
& =\int_{G} \overline{\phi \cdot \mu}(x) d m(x) \\
& =\int_{G} \overline{\phi \cdot \mu}\left(x, x^{-1}\right) d m(x) \\
& =\int_{G} \int_{G} \bar{\phi}\left(y x, x^{-1}\right) d \mu(y) d m(x) \\
& =\int_{G} \int_{G} \bar{\phi}\left(y x, x^{-1}\right) d m(x) d \mu(y) \quad \text { (by Fubini's theorem) } \\
& \left.=\int_{G} \int_{G} \bar{\phi}\left(x, x^{-1} y\right) d m(x) d \mu(y) \quad \text { (substituting } y^{-1} x \text { for } x\right) \\
& =\int_{G G} \int_{G} \bar{\phi}\left(x, x^{-1} y\right) d \mu(y) d m(x) \quad \text { (again by Fubini's theorem) } \\
& =\int_{G} \mu \cdot \phi(x) d m(x) \\
& =\langle\mu \cdot \phi, M\rangle \\
& =\langle\phi, M \cdot \mu\rangle .
\end{aligned}
$$

Thus, $M$ is a virtual $w^{*}$-diagonal for $M(G)$. 
Remarks. 1. Most of the proof of Proposition 5.2 still works for not necessarily compact, amenable $G$, where we replace Haar measure in the definition of $M$ by a left invariant mean on $L^{\infty}(G)$. In this more general situation, however, we have no substitute for the two applications (3) and (4) of Fubini's theorem.

2. The locally compact groups $G$ for which $M(G)$ is Connes amenable are characterized in the companion paper [Run 2]: They are precisely the amenable groups. In the same paper, it is also shown that $M(G)$ has a virtual $w^{*}$-diagonal if and only if it is Connes amenable.

Another important dual Banach algebra associated with a locally compact group $G$ is the group von Neumann algebra $\mathrm{VN}(G)$. Its Connes amenability was characterized in terms of $G$ by A. T.-M. Lau and A. L. T. Paterson $([\mathrm{L}-\mathrm{P}])$. There are analogues of $\mathrm{VN}(G)$ acting on $L^{p}(G)$ for $p \in(1, \infty)$, the so-called algebras of pseudo-measures $\operatorname{PM}_{p}(G)$ (for information on these algebras and further references, see [Eym]). We shall now prove an extension of [L-P, Corollary 3.3] for these algebras of pseudo-measures.

Recall (from $[\mathrm{L}-\mathrm{P}]$, for example) that a locally compact group is inner amenable if there is a state $m$ on $L^{\infty}(G)$ such that

$$
\left\langle\delta_{x} * \phi * \delta_{x^{-1}}, m\right\rangle=\langle\phi, m\rangle \quad\left(x \in G, \phi \in L^{\infty}(G)\right) .
$$

Every amenable group is inner amenable, but so is every [IN]-group.

THEOREM 5.3. For a locally compact group $G$ consider the following:

(i) $G$ is amenable.

(ii) $M(G)$ is Connes amenable.

(iii) $\mathrm{PM}_{p}(G)$ is Connes amenable for every $p \in(1, \infty)$.

(iv) $\mathrm{VN}(G)$ is Connes amenable.

(v) $\mathrm{PM}_{p}(G)$ is Connes amenable for one $p \in(1, \infty)$.

Then $(\mathrm{i}) \Rightarrow(\mathrm{ii}) \Rightarrow(\mathrm{iii}) \Rightarrow(\mathrm{iv}) \Rightarrow(\mathrm{v})$. If $G$ is inner amenable, then also $(\mathrm{v}) \Rightarrow(\mathrm{i})$.

Proof. (i) $\Rightarrow$ (ii). This is is clear in view of Proposition 4.2(i).

(ii) $\Rightarrow$ (iii). This follows from Proposition 4.2(ii).

(iii) $\Rightarrow(\mathrm{iv}) \Rightarrow(\mathrm{v})$. Since $\mathrm{VN}(G)=\mathrm{PM}_{2}(G)$, this is obvious.

$(\mathrm{v}) \Rightarrow(\mathrm{i})$ for $G$ inner amenable: For any $r \in[1, \infty)$, let $\lambda_{r}$ and $\varrho_{r}$ denote the regular left and right representations, respectively, of $G$ on $L^{r}(G)$.

By [L-R, Proposition 1], it follows from the inner amenability of $G$ that there is a net $\left(f_{\alpha}\right)_{\alpha}$ of positive $L^{1}$-functions with $\left\|f_{\alpha}\right\|_{1}=1$ such that

$$
\left\|\delta_{x} * f_{\alpha} * \delta_{x^{-1}}-f_{\alpha}\right\|_{1} \rightarrow 0 \quad(x \in G),
$$

or equivalently,

$$
\left\|\lambda_{1}\left(x^{-1}\right) f_{\alpha}-\varrho_{1}(x) f_{\alpha}\right\|_{1} \rightarrow 0 \quad(x \in G) .
$$


Let $q \in(1, \infty)$ be the index dual to $p$. Let $\xi_{\alpha}:=f_{\alpha}^{1 / p}$ and $\eta_{\alpha}:=f_{\alpha}^{1 / q}$, so that $\xi_{\alpha} \in L^{p}(G)$ and $\eta_{\alpha} \in L^{q}(G)$. It follows from (5) and [Pat, 4.3(1)] that (6) $\left\|\lambda_{p}\left(x^{-1}\right) \xi_{\alpha}-\varrho_{p}(x) \xi_{\alpha}\right\|_{p} \rightarrow 0, \quad\left\|\lambda_{q}\left(x^{-1}\right) \eta_{\alpha}-\varrho_{q}(x) \eta_{\alpha}\right\|_{q} \rightarrow 0 \quad(x \in G)$. For $\phi \in \mathrm{UC}(G)$, let $M_{\phi} \in \mathcal{L}\left(L^{p}(G)\right)$ be defined by pointwise multiplication with $\phi$. By Theorem 5.1, applied to $\mathfrak{A}=\operatorname{PM}_{p}(G), \mathfrak{B}=\mathcal{L}\left(L^{p}(G)\right)$, and $\theta$ the canonical representation of $\operatorname{PM}_{p}(G)$ on $L^{p}(G)$, there is a quasi-expectation $\mathcal{Q}: \mathcal{L}\left(L^{p}(G)\right) \rightarrow \operatorname{PM}_{p}(G)^{\prime}$. Define $m_{\alpha} \in \mathrm{UC}(G)^{*}$ by letting

$$
\left\langle\phi, m_{\alpha}\right\rangle:=\left\langle\mathcal{Q}\left(M_{\phi}\right) \xi_{\alpha}, \eta_{\alpha}\right\rangle \quad(\phi \in \mathrm{UC}(G)) .
$$

Let $\mathcal{U}$ be an ultrafilter on the index set of $\left(f_{\alpha}\right)_{\alpha}$ that dominates the order filter, and define

$$
\langle\phi, m\rangle:=\lim _{\mathcal{U}}\left\langle\phi, m_{\alpha}\right\rangle \quad(\phi \in \mathrm{UC}(G)) .
$$

Note that $\varrho_{p}(G) \subset \operatorname{PM}_{p}(G)^{\prime}$, and observe again that

$$
\varrho_{p}\left(x^{-1}\right) M_{\phi} \varrho_{p}(x)=M_{\phi * \delta_{x}} \quad(x \in G, \phi \in \mathrm{UC}(G)) .
$$

We then obtain for $x \in G$ and $\phi \in \mathrm{UC}(G)$ :

$$
\begin{aligned}
\left\langle\phi * \delta_{x}, m\right\rangle & =\lim _{\mathcal{U}}\left\langle\phi * \delta_{x}, m_{\alpha}\right\rangle \\
& =\lim _{\mathcal{U}}\left\langle\mathcal{Q}\left(\varrho_{p}\left(x^{-1}\right) M_{\phi} \varrho_{p}(x)\right) \xi_{\alpha}, \eta_{\alpha}\right\rangle \\
& =\lim _{\mathcal{U}}\left\langle\varrho_{p}\left(x^{-1}\right)\left(\mathcal{Q} M_{\phi}\right) \varrho_{p}(x) \xi_{\alpha}, \eta_{\alpha}\right\rangle \\
& =\lim _{\mathcal{U}}\left\langle\left(\mathcal{Q} M_{\phi}\right) \varrho_{p}(x) \xi_{\alpha}, \varrho_{p}(x) \eta_{\alpha}\right\rangle \\
& =\lim _{\mathcal{U}}\left\langle\left(\mathcal{Q} M_{\phi}\right) \lambda_{p}\left(x^{-1}\right) \xi_{\alpha}, \lambda_{p}\left(x^{-1}\right) \eta_{\alpha}\right\rangle \\
& \left.=\lim _{\mathcal{U}}\left\langle\lambda_{p}(x) \mathcal{Q} M_{\phi}\right) \lambda_{p}\left(x^{-1}\right) \xi_{\alpha}, \eta_{\alpha}\right\rangle \\
& =\lim _{\mathcal{U}}\left\langle\left(\mathcal{Q} M_{\phi}\right) \xi_{\alpha}, \eta_{\alpha}\right\rangle \\
& =\langle\phi, m\rangle .
\end{aligned}
$$

Hence, $m$ is right invariant. Clearly, $\langle 1, m\rangle=1$. Taking the positive part of $m$ and normalizing it, we obtain a right invariant mean on $\mathrm{UC}(G)$.

Remarks. 1 . The hypothesis that $G$ be inner amenable cannot be dropped: As pointed out in [Pat, p. 84], $\mathrm{SL}(2, \mathbb{R})$ is not amenable, but of type I, so that $\operatorname{VN}(\mathrm{SL}(2, \mathbb{R}))$ is Connes amenable.

2. In $[\mathrm{L}-\mathrm{P}]$, Lau and Paterson show that an inner amenable, locally compact group $G$ such that $\mathrm{VN}(G)$ has Schwartz' property $(\mathrm{P})$ is already amenable. Schwartz' property $(\mathrm{P})$ and Connes amenability are equivalent (see [Pat, (2.35)]), but the implication from Connes amenability to $(\mathrm{P})$ is a deep result by itself. The proof of Theorem 5.3 is thus much simpler, even in the particular case $p=2$. 
6. A nuclear-free characterization of amenable $W^{*}$-algebras. As we have mentioned in the introduction, the following characterization of the amenable $W^{*}$-algebras is known:

TheOrem 6.1. For $a W^{*}$-algebra $\mathfrak{A}$, the following are equivalent:

(i) $\mathfrak{A}$ is amenable.

(ii) There are hyperstonean, compact spaces $\Omega_{1}, \ldots, \Omega_{n}$ and $n_{1}, \ldots, n_{k} \in$ $\mathbb{N}$ such that

$$
\mathfrak{A} \cong \bigoplus_{j=1}^{k} \mathbb{M}_{n_{j}} \otimes \mathcal{C}\left(\Omega_{j}\right) .
$$

The implication (ii) $\Rightarrow$ (i) is obvious, and the converse is a consequence of [Con 2], [Was, Corollary (1.9)], and the structure theory of $W^{*}$-algebras.

In this section, we give a proof of Theorem 6.1 which avoids the amenability-nuclearity nexus and only relies on standard facts about $W^{*}$-algebras, where we define a standard fact as one that can be found in one of the standard books on the subject such as [Dix], [K-R], [Sak], and [Tak].

In analogy with [Was], we define:

Definition 6.2. Let $\mathfrak{A}$ be a Banach *-algebra. We say that $\mathfrak{A}$ is of type $(\mathrm{QE})$ if, for each ${ }^{*}$-representation $(\pi, \mathfrak{H})$, there is a quasi-expectation $\mathcal{Q}: \mathcal{B}(\mathfrak{H}) \rightarrow \pi(\mathfrak{A})^{\prime \prime}$

REMARK. It follows from [B-P] that every $C^{*}$-algebra which is of type (QE) is already of type (E) in the sense of [Was]. However, since we strive to keep the $W^{*}$-theory required for the proof of Theorem 6.1 to a minimum, we shall not use this fact.

The next two results are essentially already contained in [Tom]. Here we give proofs requiring a minimum of $W^{*}$-theory.

Lemma 6.3. Let $\mathfrak{A}$ be a von Neumann algebra acting on a Hilbert space $\mathfrak{H}$. Then the following are equivalent:

(i) There is a quasi-expectation $\mathcal{Q}: \mathcal{B}(\mathfrak{H}) \rightarrow \mathfrak{A}$.

(ii) For every faithful normal representation $(\pi, \mathfrak{K})$ of $\mathfrak{A}$, there is a quasiexpectation $\mathcal{Q}: \mathcal{B}(\mathfrak{K}) \rightarrow \pi(\mathfrak{A})^{\prime \prime}$.

Proof. Let $\pi$ be a faithful $W^{*}$-representation of $\mathfrak{M}$ on a Hilbert space $\mathfrak{K}$, and let $\mathfrak{N}:=\pi(\mathfrak{M})$. Using the idea of the proof of [Dix, Théorème 3, §I.4], we can choose a third Hilbert space $\mathfrak{L}$ such that $\mathfrak{M} \otimes \mathrm{id}_{\mathfrak{L}}$, i.e. the algebra $\left\{x \otimes \operatorname{id}_{\mathfrak{L}}: x \in \mathfrak{M}\right\}$, on $\mathfrak{H} \bar{\otimes} \mathfrak{L}$ and $\mathfrak{N} \otimes \mathrm{id}_{\mathfrak{L}}$ on $\mathfrak{K} \bar{\otimes} \mathfrak{L}$ are spatially isomorphic. Fix $\xi_{0}, \eta_{0} \in \mathfrak{L}$ such that $\left\langle\xi_{0}, \eta_{0}\right\rangle=1$, and define $\mathcal{P}_{0}: \mathcal{L}(\mathfrak{H} \bar{\otimes} \mathfrak{L}) \rightarrow \mathcal{L}(\mathfrak{H})$ through

$$
\left\langle\left(\mathcal{P}_{0} T\right) \xi, \eta\right\rangle:=\left\langle T\left(\xi \otimes \xi_{0}\right), \eta \otimes \eta_{0}\right\rangle \quad(T \in \mathcal{L}(\mathfrak{H}), \xi, \eta \in \mathfrak{H}) .
$$


Identifying $\mathcal{L}(\mathfrak{H})$ with $\mathcal{L}(\mathfrak{H}) \otimes$ id $_{\mathfrak{L}}$, we see that $\mathcal{P}_{0}$ is a projection onto $\mathcal{L}(\mathfrak{H})$. Furthermore, for $T \in \mathcal{L}(\mathfrak{H} \bar{\otimes} \mathfrak{L})$ and $R, S \in \mathcal{L}(\mathfrak{H})$, we have

$$
\begin{aligned}
\left.\mathcal{P}_{0}\left(\left(R \otimes \operatorname{id}_{\mathfrak{L}}\right) T\left(S \otimes \operatorname{id}_{\mathfrak{L}}\right)\right) \xi, \eta\right\rangle & =\left\langle\left(R \otimes \operatorname{id}_{\mathfrak{L}}\right) T\left(S \otimes \operatorname{id}_{\mathfrak{L}}\right)\left(\xi \otimes \xi_{0}\right), \eta \otimes \eta_{0}\right\rangle \\
& =\left\langle T\left(S \xi \otimes \xi_{0}\right), R^{*} \eta \otimes \eta_{0}\right\rangle \\
& =\left\langle\left(\mathcal{P}_{0} T\right) S \xi, R^{*} \eta\right\rangle \\
& =\left\langle R\left(\mathcal{P}_{0} T\right) S \xi, \eta\right\rangle \quad(\xi, \eta \in \mathfrak{H}),
\end{aligned}
$$

so that $\mathcal{P}_{0}$ is a quasi-expectation. Let $\mathcal{P}: \mathcal{L}(\mathfrak{H}) \rightarrow \mathfrak{M}$ be a quasi-expectation, and define

$$
\widetilde{\mathcal{P}}: \mathcal{L}(\mathfrak{H} \bar{\otimes} \mathfrak{L}) \rightarrow \mathfrak{M} \otimes \operatorname{id}_{\mathfrak{L}}, \quad T \mapsto\left(\mathcal{P} \circ \mathcal{P}_{0}\right) T \otimes \operatorname{id}_{\mathfrak{L}} ;
$$

it is clear that $\widetilde{\mathcal{P}}$ is also a quasi-expectation. Since $\mathfrak{M} \otimes \mathrm{id}_{\mathfrak{L}}$ and $\mathfrak{N} \otimes \mathrm{id}_{\mathfrak{L}}$ are spatially isomorphic, there is a quasi-expectation $\widetilde{\mathcal{Q}}: \mathcal{L}(\mathfrak{K} \bar{\otimes} \mathfrak{L}) \rightarrow \mathfrak{N} \otimes \mathrm{id}_{\mathfrak{L}}$. Fix again $\xi_{0}, \eta_{0} \in \mathfrak{L}$ such that $\left\langle\xi_{0}, \eta_{0}\right\rangle=1$, and define $\mathcal{Q}_{0}: \mathcal{L}(\mathfrak{K} \bar{\otimes} \mathfrak{L}) \rightarrow \mathcal{L}(\mathfrak{K})$ as in (7). Then

$$
\mathcal{Q}: \mathcal{L}(\mathfrak{K}) \rightarrow \mathfrak{N}, \quad T \mapsto\left(\mathcal{Q}_{0} \circ \widetilde{\mathcal{Q}}\right)\left(T \otimes \mathrm{id}_{\mathfrak{L}}\right),
$$

is the desired quasi-expectation.

Proposition 6.4. Every amenable Banach ${ }^{*}$-algebra is of type (QE).

Proof. Let $(\pi, \mathfrak{H})$ be a ${ }^{*}$-representation of $\mathfrak{A}$. Then, by $[\mathrm{K}-\mathrm{R}$, Exercise 7.6.46], there is a faithful normal, semifinite weight on the von Neumann algebra $\pi(\mathfrak{A})^{\prime \prime}$. Let $(\varrho, \mathfrak{K})$ be the faithful normal representation of $\pi(\mathfrak{A})^{\prime \prime}$ constructed from this weight ([K-R, Theorem 7.5.3]). By [C-G 2, Proposition 2.2 ], applied to the representation $(\varrho \circ \pi, \mathfrak{K})$ of $\mathfrak{A}$, there is a quasi-expectation $\mathcal{P}: \mathcal{B}(\mathfrak{K}) \rightarrow(\varrho \circ \pi)(\mathfrak{A})^{\prime}$. Let $J: \mathfrak{K} \rightarrow \mathfrak{K}$ be the conjugate linear isometry from $[\mathrm{K}-\mathrm{R}$, Theorem 9.2.37], i.e.

$$
J^{2}=\operatorname{id}_{\mathfrak{K}} \quad \text { and } \quad J(\varrho \circ \pi)(\mathfrak{A})^{\prime} J=(\varrho \circ \pi)(\mathfrak{A})^{\prime \prime} .
$$

Define

$$
\widetilde{\mathcal{Q}}: \mathcal{B}(\mathfrak{K}) \rightarrow(\varrho \circ \pi)(\mathfrak{A})^{\prime \prime}, \quad a \mapsto J \mathcal{P}(J a J) J .
$$

It is immediate that $\widetilde{\mathcal{Q}}$ is a quasi-expectation. Since $\varrho$ is normal and faithful, it follows from Lemma 6.3 that there is a quasi-expectation $\mathcal{Q}: \mathcal{B}(\mathfrak{H}) \rightarrow$ $\pi(\mathfrak{A})^{\prime \prime}$.

Our next lemma is an analogue of [Was, Proposition (1.2)], whose proof carries over almost verbatim:

Lemma 6.5. Let $\mathfrak{A}$ be a $C^{*}$-algebra of type (QE), and let $\mathfrak{B}$ be a $C^{*}$-algebra such that there is a quasi-expectation $\mathcal{Q}: \mathfrak{A} \rightarrow \mathfrak{B}$. Then $\mathfrak{B}$ is of type $(\mathrm{QE})$.

Also, the following proposition is well known: It is an immediate consequence of [L-P, Corollary 3.3], [Tom, Theorem 7.2], and [B-P, Theo- 
rem 2]. We prefer, however, to indicate a proof that completely avoids $W^{*}$ theoretical arguments and uses ideas from $[\mathrm{C}-\mathrm{G} 2]$ instead:

Proposition 6.6. For an inner amenable group $G$, the following are equivalent:

(i) $G$ is amenable.

(ii) There is a quasi-expectation $\mathcal{Q}: \mathcal{B}\left(L^{2}(G)\right) \rightarrow \mathrm{VN}(G)$.

Proof. For the proof of (i) $\Rightarrow\left(\right.$ ii) note that $\mathrm{VN}(G)=\varrho_{2}(G)^{\prime}$. An application of $[\mathrm{C}-\mathrm{G} 2$, Proposition 2.2] then yields the claim.

For the converse implication, we proceed as in the proof of Theorem 5.3 , with the rôles of $\lambda_{2}$ and $\varrho_{2}$ interchanged, and obtain a left invariant $m \in \mathrm{UC}(G)^{*}$ with $\langle 1, m\rangle=1$.

As in [Was], we obtain:

Corollary 6.7. The $W^{*}$-algebras $\mathrm{VN}\left(\mathbb{F}_{2}\right)$ and $\mathbb{M}_{\infty}$ are not of type (QE) and thus, in particular, are not amenable.

Proof. Since $\mathbb{F}_{2}$ is well known (and easily seen) to be non-amenable ([Pat, Example (0.6)]), there is no quasi-expectation $\mathcal{Q}: \mathcal{B}\left(\ell^{2}\left(\mathbb{F}_{2}\right)\right) \rightarrow \mathrm{VN}\left(\mathbb{F}_{2}\right)$ by Proposition 6.6.

The case of $\mathbb{M}_{\infty}$ is reduced to $\mathrm{VN}\left(\mathbb{F}_{2}\right)$ as in [Was]: We can find a maximal ideal $M$ of $\mathbb{M}_{\infty}$ corresponding to a point in $\beta \mathbb{N} \backslash \mathbb{N}$ such that $\mathfrak{A}:=\mathbb{M}_{\infty} / M$ contains a $W^{*}$-subalgebra $\mathfrak{B}$ which is isomorphic to $\mathrm{VN}\left(\mathbb{F}_{2}\right)$. As pointed out in [Tak, pp. 358-359], $\mathfrak{A}$ is a type $\mathrm{II}_{1}$ factor, which by [Sak, 4.4.23 Proposition] means that there is a (norm one) quasi-expectation $\mathcal{Q}: \mathfrak{A} \rightarrow \mathfrak{B}$. Hence, if $\mathbb{M}_{\infty}$ were of type (QE), the same would be true for $\mathfrak{A}$ and, by Lemma 6.5 , for $\mathfrak{B} \cong \mathrm{VN}\left(\mathbb{F}_{2}\right)$. However, as we have just seen, $\mathrm{VN}\left(\mathbb{F}_{2}\right)$ fails to be of type $(\mathrm{QE})$.

REMARK. Although the proof for the non-amenability of $\mathbb{M}_{\infty}$ given here is more elementary than the one from $[\mathrm{L}-\mathrm{L}-\mathrm{W}]$, it is not yet as satisfying as we would like it to be: The proof of Proposition 6.4 relies on TomitaTakesaki theory, which still seems to be far too deep in order to prove a result on an algebra as plain as $\mathbb{M}_{\infty}$. We could have avoided the use of Tomita-Takesaki theory in the proof of Proposition 6.4 by defining type (QE) via $\pi(\mathfrak{A})^{\prime}$ instead of $\pi(\mathfrak{A})^{\prime \prime}$; Proposition 6.4 would then have been a straightforward application of [C-G 2, Proposition 2.2]. Then, however, we would have required Tomita-Takesaki theory in the proof of Lemma 6.5.

Proof of Theorem 6.1. Suppose that $\mathfrak{A}$ is not of the form in Theorem 6.1(ii). Then, just as in [Was], it follows from the general structure theory of $W^{*}$-algebras that $\mathfrak{A}$ contains $\mathbb{M}_{\infty}$ as a closed subalgebra. As in the proof of [Was, Corollary (1.8)], a norm one projection $\mathcal{Q}: \mathfrak{A} \rightarrow \mathbb{M}_{\infty}$ can be 
constructed, which is easily, i.e. directly and without [Tom, Theorem 3.1], seen to be a quasi-expectation.

The proof of Theorem 6.1 would be considerably easier if we could show by elementary means that, for an amenable $C^{*}$-algebra $\mathfrak{A}$, a $C^{*}$-subalgebra $\mathfrak{B}$, and a quasi-expectation $\mathcal{Q}: \mathfrak{A} \rightarrow \mathfrak{B}$, the algebra $\mathfrak{B}$ is amenable as well. This is indeed true, but in order to prove it we need the deep connections between amenability, nuclearity and injectivity.

The corresponding claim for general Banach algebras is false:

EXAMPLE. Let $G$ be a compact group. Then $L^{1}(G)$ is amenable, so that $L^{1}(G)$ has an approximate diagonal $\left(m_{\alpha}\right)_{\alpha}$. Let $\mathcal{U}$ be an ultrafilter on the index set of $\left(d_{\alpha}\right)_{\alpha}$ which dominates the order filter, and define

$$
\mathcal{Q}: L^{1}(G) \rightarrow Z\left(L^{1}(G)\right), \quad f \mapsto \lim _{\mathcal{U}} m_{\alpha} \cdot f
$$

where

$$
(g \otimes h) \cdot f:=g * f * h \quad\left(f, g, h \in L^{1}(G)\right)
$$

and the limit is taken in the norm topology; since multiplication by any element of $L^{1}(G)$ is compact, this limit does indeed exist. It is clear that $\mathcal{Q}$ is a quasi-expectation. However, there are compact groups $G$ such that $Z\left(L^{1}(G)\right)$ is not amenable ([Ste]).

\section{References}

[B-M] G. Brown and W. Moran, Point derivations on $M(G)$, Bull. London Math. Soc. 8 (1976), 57-64.

[B-P] J. W. Bunce and W. L. Paschke, Quasi-expectations and amenable von Neumann algebras, Proc. Amer. Math. Soc. 71 (1978), 232-236.

[Con 1] A. Connes, Classification of injective factors, Ann. of Math. 104 (1976), 73114.

[Con 2] -, On the cohomology of operator algebras, J. Funct. Anal. 28 (1978), 248-253.

[C-G 1] G. Corach and J. E. Galé, Averaging with virtual diagonals and geometry of representations, in: E. Albrecht and M. Mathieu (eds.), Banach Algebras '97, de Gruyter, 1998, 87-100.

[C-G 2] - - - On amenability and geometry of spaces of bounded representations, J. London Math. Soc. (2) 59 (1999), 311-329.

[C-L] P. C. Curtis, Jr., and R. J. Loy, The structure of amenable Banach algebras, ibid. 40 (1989), 89-104.

[D-G-H] H. G. Dales, F. Ghahramani and A. Ya. Helemskiur, The amenability of measure algebras, preprint, 2001.

[D-F] A. Defant and K. Floret, Tensor Norms and Operator Ideals, North-Holland, 1993.

[Dix] J. Dixmier, Les algèbres d'opérateurs dans l'espace hilbertien (algèbres de von Neumann), 2nd ed., Gauthier-Villars, 1969.

[D-ت̈] J. Duncan and A. Ülger, Almost periodic functionals on Banach algebras, Rocky Mountain J. Math. 22 (1992), 837-848. 
[Eff] E. G. Effros, Amenability and virtual diagonals for von Neumann algebras, J. Funct. Anal. 78 (1988), 137-153.

[E-K] E. G. Effros and A. Kishimoto, Module maps and Hochschild-Johnson cohomology, Indiana Univ. Math. J. 36 (1987), 257-276.

[Eym] P. Eymard, Algèbres $A_{p}$ et convoluteurs de $L^{p}$, in: Séminaire Bourbaki 1969/70, no. 367, Springer, 1971, 55-72.

[G-R-W] J. E. Galé, T. J. Ransford and M. C. White, Weakly compact homomorphisms, Trans. Amer. Math. Soc. 331 (1992), 815-824.

[G-L-W] F. Ghahramani, R. J. Loy and G. A. Willis, Amenability and weak amenability of second conjugate Banach algebras, Proc. Amer. Math. Soc. 124 (1996), 1489-1497.

[Gou] F. Gourdeau, Amenability and the second dual of a Banach algebra, Studia Math. 125 (1997), 75-81.

[Grø] N. Grønbæk, Various notions of amenability, a survey of problems, in: E. Albrecht and M. Mathieu (eds.), Banach Algebras '97, de Gruyter, 1998, 535548 .

[G-J-W] N. Grønbæk, B. E. Johnson and G. A. Willis, Amenability of Banach algebras of compact operators, Israel J. Math. 87 (1994), 289-324.

[Gro] M. Grosser, Bidualräume und Vervollständigungen von Banachmoduln, Springer, 1979.

[Haa] U. Haagerup, All nuclear $C^{*}$-algebras are amenable, Invent. Math. 74 (1983), 305-319.

[Hel 1] A. Ya. Helemskiı̌, The Homology of Banach and Topological Algebras, Kluwer, 1989 (translated from Russian).

[Hel 2] -, Some remarks about ideas and results of topological homology, in: R. J. Loy (ed.), Conference on Automatic Continuity and Banach Algebras, Austral. Nat. Univ., 1989, 203-238..

[Joh 1] B. E. Johnson, Separate continuity and measurability, Proc. Amer. Math. Soc. 20 (1969), 420-422.

[Joh 2] - Cohomology in Banach algebras, Mem. Amer. Math. Soc. 127 (1972).

[Joh 3] - Approximate diagonals and cohomology of certain annihilator Banach algebras, Amer. J. Math. 94 (1972), 685-698.

[J-K-R] B. E. Johson, R. V. Kadison and J. Ringrose, Cohomology of operator algebras, III, Bull. Soc. Math. France 100 (1972), 73-79.

[K-R] R. V. Kadison and J. R. Ringrose, Fundamentals of the Theory of Operator Algebras, II, Academic Press, 1986.

[L-L] A. T.-M. Lau and R. J. Loy, Amenability of convolution algebras, Math. Scand. 79 (1996), 283-296.

[L-L-W] A. T.-M. Lau, R. J. Loy and G. A. Willis, Amenability of Banach and $C^{*}$ algebras on locally compact groups, Studia Math. 119 (1996), 161-178.

[L-P] A. T.-M. Lau and A. L. T. Paterson, Inner amenable locally compact groups, Trans. Amer. Math. Soc. 325 (1991), 155-169.

[L-R] V. Losert and H. Rindler, Conjugate invariant means, Colloq. Math. 51 (1987), 221-225.

[Pal 1] T. W. Palmer, Arens multiplication and a characterization of $W^{*}$-algebras, Proc. Amer. Math. Soc. 44 (1974), 81-87.

[Pal 2] - Banach Algebras and the General Theory of ${ }^{*}$-Algebras, I, Cambridge Univ. Press, 1994.

[Pat] A. L. T. Paterson, Amenability, Amer. Math. Soc., 1988. 
[Rea] C. J. Read, Commutative, radical amenable Banach algebras, Studia Math. 140 (2000), 199-212.

[Run 1] V. Runde, The structure of contractible and amenable Banach algebras, in: E. Albrecht and M. Mathieu (eds.), Banach Algebras '97, de Gruyter, 1998, 415-430.

[Run 2] - Connes-amenability and normal, virtual diagonals for measure algebras, J. London Math. Soc., to appear.

[Sak] S. Sakai, $C^{*}$-Algebras and $W^{*}$-Algebras, Springer, 1971.

[Sel] Yu. V. Selivanov, On Banach algebras of small global dimension zero, Uspekhi Mat. Nauk 31 (1976), no. 2, 227-228 (in Russian).

[She] M. V. Shel̆nberg, A characterization of the algebra $C(\Omega)$ in terms of cohomology groups, ibid. 32 (1977), no. 5, 203-204 (in Russian).

[Ste] U. Stegmeir, Centers of group algebras, Math. Ann. 243 (1979), 11-16.

[Tak] M. Takesaki, Theory of Operator Algebras, I, Springer, 1979.

[Tom] J. Tomiyama, Tensor products and projections of norm one in von Neumann algebras, lecture notes, Univ. of Copenhagen, 1970.

[Ülg] A. Ülger, Arens regularity of $K(X)$, Monatsh. Math. 105 (1988), 313-318.

[Was] S. Wassermann, On tensor products of certain group $C^{*}$-algebras, J. Funct. Anal. 23 (1976), 239-254.

Department of Mathematical Sciences

University of Alberta

Edmonton, Alberta

Canada T6G 2G1

E-mail: runde@math.ualberta.ca

vrunde@ualberta.ca 